\title{
Universal Bifurcations and the Classification of Cardiac Arrhythmias ${ }^{a}$
}

\author{
LEON GLASS, MICHAEL R. GUEVARA, \\ AND ALVIN SHRIER \\ Department of Physiology \\ McGill University \\ Montreal, Quebec, Canada H3G 1 Y6
}

\section{INTRODUCTION}

An appreciation of the extraordinarily rich dynamics of cardiac arrhythmias can be obtained from examination of any text in electrocardiography. Typically, there are a large number (100 or more) of rhythms that are identified on the basis of changes outside of normal limits of rhythm and wave morphology in the electrocardiogram. ${ }^{1,2}$ The point of this paper is to show that mathematics can provide a framework for studying several cardiac arrhythmias. It is possible to theoretically predict dynamics in some biological models and clinical situations using appropriate mathematical models.

The main theoretical technique used is to represent cardiac systems by a finitedifference equation of the form,

$$
x_{t+1}=f\left(x_{t}, \lambda\right),
$$

where $f$ is a nonlinear function, $x_{t}$ is the value of a variable measured at discrete time $t$, and $\lambda$ is a parameter. ${ }^{3}$ Once an initial condition $x_{0}$ is chosen, and provided the function $f$ is known, the sequence $x_{1}, x_{2}, \ldots$ can be generated by an iterative procedure. Even for simple nonlinear functions $f$, the possible range of dynamics includes equilibria, stable periodic cycles, and "chaos" (aperiodic dynamics in a deterministic system demonstrating sensitive dependence on initial conditions). A qualitative change in the dynamics due to a change in the parameter $\lambda$ is called a bifurcation. $A$ remarkable observation is that for simple classes of nonlinear functions, the same sequences of bifurcations are observed for all members of that class as $\lambda$ is changed. Thus, the bifurcations are called universal. ${ }^{3-5}$

In what follows, we examine the bifurcations present in finite-difference equations that model cardiac systems and compare the predicted dynamics with experimental observations. First, we review previous studies of certain cardiac arrhythmias. Then,

\footnotetext{
${ }^{a}$ This work was supported by grants from the Canadian Heart Foundation (CHF) and the Natural Sciences and Engineering Research Council of Canada (NSERC). M.R.G. was the recipient of a predoctoral fellowship from the CHF and postdoctoral fellowships from CHF and NSERC. During 1984-86, M.R.G. was a postdoctoral fellow in the Fysiologisch Laboratorium, Universiteit van Amsterdam, Amsterdam, The Netherlands. During the 1984-85 academic year, L.G. was a visiting scientist at the Institute for Nonlinear Sciences, University of California at San Diego, La Jolla, California.
} 
we show how the response of cardiac tissue to periodic stimulation can be modeled by finite-difference equations. After that, we discuss the periodic stimulation of nonoscillating, but excitable cardiac tissue and also of spontaneously oscillating cardiac tissue. Finally, we show that these results can be used to develop a partial classification of cardiac arrhythmias and we discuss the limitations of this approach.

\section{MECHANISMS OF CARDIAC ARRHYTHMIAS}

The intrinsic rhythm of the normal heart is set by a small region of specialized cells located in the right atrium called the sinoatrial (SA) node. From the SA node, the cardiac impulse spreads sequentially through the atrial musculature, the atrioventricular (AV) node, and then through the bundle of His, the bundle branches, and specialized conducting tissue called the Purkinje fibers to the ventricular muscle. Cardiac arrhythmias are often associated with abnormalities in the generation of the spontaneous rhythm at the SA node, abnormal propagation of the cardiac activity, or development of spontaneous rhythmic activity at abnormal locations (ectopic foci). These abnormalities can arise at virtually any location in the heart and can occur in combination, thus leading to a large number of different cardiac arrhythmias that are frequently complex and difficult to interpret. ${ }^{1,2}$ To simplify matters, we discuss separately certain classes of arrhythmias that may be due to abnormal impulse conduction and abnormal impulse generation.

Arrhythmias due to disturbances in impulse conduction are often studied by evaluating the conduction velocity as stimulation parameters are varied. Early in this century, investigators measured the time between atrial and ventricular activation as a function of atrial frequency. It was observed that as atrial frequency is increased, the propagation time through the $\mathrm{AV}$ node is increased, and that at high atrial frequencies some of the impulses are not conducted through the AV node to the ventricles. ${ }^{6,7}$ This can lead to rhythms in which there is a progressive prolongation of the time between atrial and ventricular activations until a ventricular beat is dropped (the Wenckebach phenomenon). At very high frequencies, more than one consecutive impulse is not conducted (high-grade block). These early findings have been confirmed more recently in a number of different studies. ${ }^{8-10}$ A simple theoretical model for these results was proposed in 1924 by Mobitz." If it is assumed that the time from atrial stimulation to ventricular activity is a function (called the recovery curve) of the time that has elapsed since the preceding ventricular activation, then once the recovery curve is determined, the effects of periodic stimulation at any frequency can be calculated using an iterative procedure. ${ }^{9,12,13}$ The form of the recovery curve is generally quite simple and this allows for a detailed mathematical description of the dynamics as stimulation frequency is varied. ${ }^{14}$

Disorders related to abnormal cardiac impulse generation are often studied by considering the effects of a single stimulus or of a periodic train of stimuli delivered to spontaneously oscillating cardiac tissue. ${ }^{15}$ It has been shown that periodic stimulation of spontaneously oscillating cardiac tissue will lead to different patterns of interaction between the periodic stimulator and the spontaneous oscillator. For example, an in vitro preparation of periodically stimulated spontaneously active Purkinje fibers can be a biologic model of parasystolic rhythms (in which a ventricular ectopic focus competes 
with the normal output from the SA node). ${ }^{15-17}$ An early theoretical study of the competition between spontaneously oscillating cardiac sites was undertaken by van der Pol and van der Mark in $1928,{ }^{18}$ who modeled the heart by three coupled nonlinear electrical oscillators. More recent studies have extended this work by carrying out studies on analogue systems ${ }^{\mathbf{1 9 . 2 0}}$ and by numerical integration of nonlinear equations. ${ }^{21}$ In this work, there is continuous interaction between the oscillators, and such systems are extremely difficult to analyze mathematically. ${ }^{22}$ An approach that largely sidesteps this complication is to consider the effect of pulsatile stimulation on a single oscillator. Once the effects of a single pulse on resetting the rhythm are known, then (provided there is rapid relaxation to the cycle following a single pulse) an iterative procedure can be used to compute the effects of a periodic train of pulses. This iterative procedure was first used in studies of the circadian rhythm and periodic forcing of nerve cells. ${ }^{23,24}$ Essentially equivalent procedures were subsequently employed by many other authors. $^{15,25-28}$
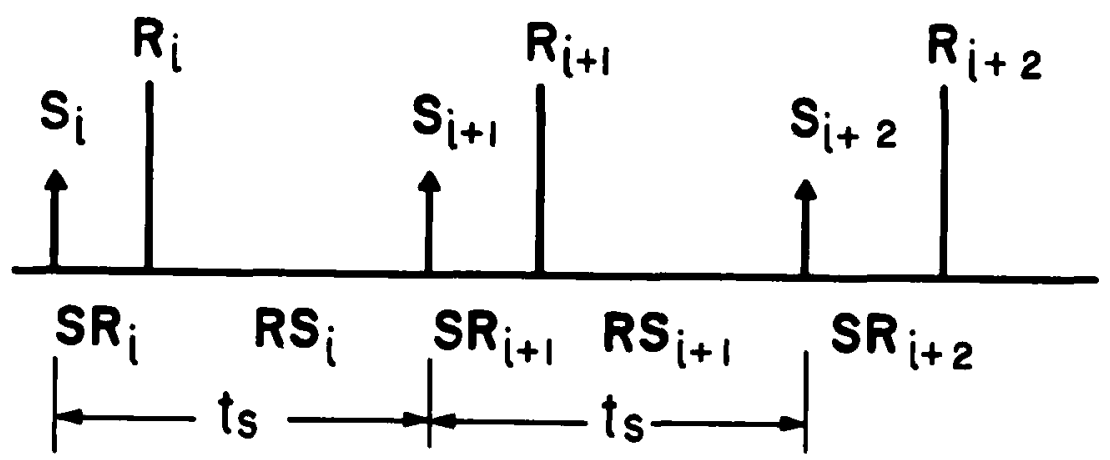

FIGURE 1. Schematic representation of experiments in which cardiac tissue is periodically stimulated. The stimulus $(S)$ is periodic in time. The response $(R)$ may or may not be measured at the same location where the stimulus is delivered.

In summary, experimental studies have shown that periodic stimulation of excitable cardiac tissue (spontaneously oscillating or not) reproduces rhythms analogous to many cardiac arrhythmias. Under certain well-defined approximations, theoretical analysis of such systems is possible using iterative procedures. We now show that such iterative procedures can be represented mathematically by finitedifference equations.

\section{CARDIAC DYNAMICS AND FINITE-DIFFERENCE EQUATIONS}

In order to cast cardiac dynamics into the form of finite-difference equations, we consider the effects of periodic stimulation of cardiac tissue, schematically represented in FIGURE 1. Assume that each stimulus (S) periodically delivered to the cardiac tissue is followed by a response (R) at some later time. One assumption that we make to facilitate mathematical analysis is that the time between the stimulus and response 
(SR) depends on the interval from the preceding response to the stimulus (RS). Referring to FIgURE 1, we write

$$
\mathrm{SR}_{i+1}=g\left(\mathrm{RS}_{i}\right)
$$

where $\mathbf{S R}_{i+1}$ is the time between the stimulus and subsequent response and $\mathbf{R S}_{i}$ is the time from preceding response to the stimulus. Equation 1 can be rewritten as

$$
\mathrm{SR}_{i+1}=g\left(t_{s}-\mathrm{SR}_{i}\right),
$$

where $t_{s}$ is the time interval between periodic stimuli, or alternatively

$$
\mathrm{RS}_{i+1}=t_{s}-\mathrm{g}\left(\mathrm{RS}_{i}\right)
$$

by using the identities $t_{s}=\mathbf{S R}_{i}+\mathbf{R S}_{i}=\mathrm{SR}_{i+1}+\mathrm{RS}_{i+1}$. Equations $3 \mathrm{a}$ and $3 \mathrm{~b}$ are finite-difference equations. We now discuss the application of these equations in two different contexts.

\section{PERIODIC STIMULATION OF NONOSCILLATING, BUT EXCITABLE CARDIAC TISSUE}

We first consider how finite-difference equations can be used to describe the conduction time of the cardiac impulse through the AV node. Using catheters inserted into the heart, data can be collected that provides information about electrical activity in the atrium, in the bundle of His (which lies just below the AV node), and in the ventricle. In a study in humans, Levy et al ${ }^{9}$ found that during periodic stimulation of the atria, at a stimulation period of $440 \mathrm{~ms}$, Wenckebach block was established in which there were 11 stimuli to 10 ventricular contractions (11:10 AV block) (FIGURE $2 \mathrm{a})$. The time intervals from a given stimulus $(\mathrm{St})$ to activity in the bundle of $\mathrm{His}(\mathrm{H})$ are also shown in Figure 2a. We designate the time interval from $\mathrm{St}$ to $\mathrm{H}$ as $\mathrm{SH}$ and that from $\mathrm{H}$ to $\mathrm{St}$ as HS. Successive measurements of $\mathrm{SH}$ as a function of the preceding $\mathrm{HS}$ interval are shown by the triangular symbols in FigurE 2b. An exponential function can be fitted to the data

$$
\mathrm{SH}=\mathrm{SH}_{\min }+\alpha \exp (-\mathrm{HS} / \tau) \quad \text { for } \quad \mathrm{HS}>\theta,
$$

where $\mathrm{SH}_{\min }=230 \mathrm{~ms}, \alpha=308 \mathrm{~ms}, \tau=111 \mathrm{~ms}$, and $\theta=50 \mathrm{~ms}(\theta$ is called the refractory period). Assume that the curve shown in FIGURE $2 \mathrm{~b}$ gives the response of the AV node during periodic stimulation of the atrium with period $t_{s}$. Let $\mathrm{SH}_{i}$ represent the $\mathrm{SH}$ interval of the $i$-th conducted impulse. By substituting Eq. 4 into Eq. 2, we obtain

$$
\mathrm{SH}_{i+1}=\mathrm{SH}_{\min }+\alpha \exp \left[-\left(N t_{s}-\mathrm{SH}_{i}\right) / \tau\right],
$$

where $N$ is the smallest integer such that $N t_{s}-\mathrm{SH}_{i}>\theta$.

By iterating Eq. 5 , it is possible to determine the dynamics for any value of $t_{s}$. The iteration may be done graphically as illustrated in FIGURE 2c, in which $\mathrm{SH}_{i+1}$ is plotted as a function of $\mathrm{SH}_{i}$ using Eq. 5 with $t_{s}=442 \mathrm{~ms}$. The resulting cycle corresponds to 10:9 AV block. Alternately, Eq. 5 can be numerically iterated on a digital computer. The results are shown in Figure $2 d$, which gives the conduction ratio $\rho$ ( $\rho$ is the average number of ventricular contractions per atrial stimulus, i.e., for $N: M$ block, $\rho=$ 

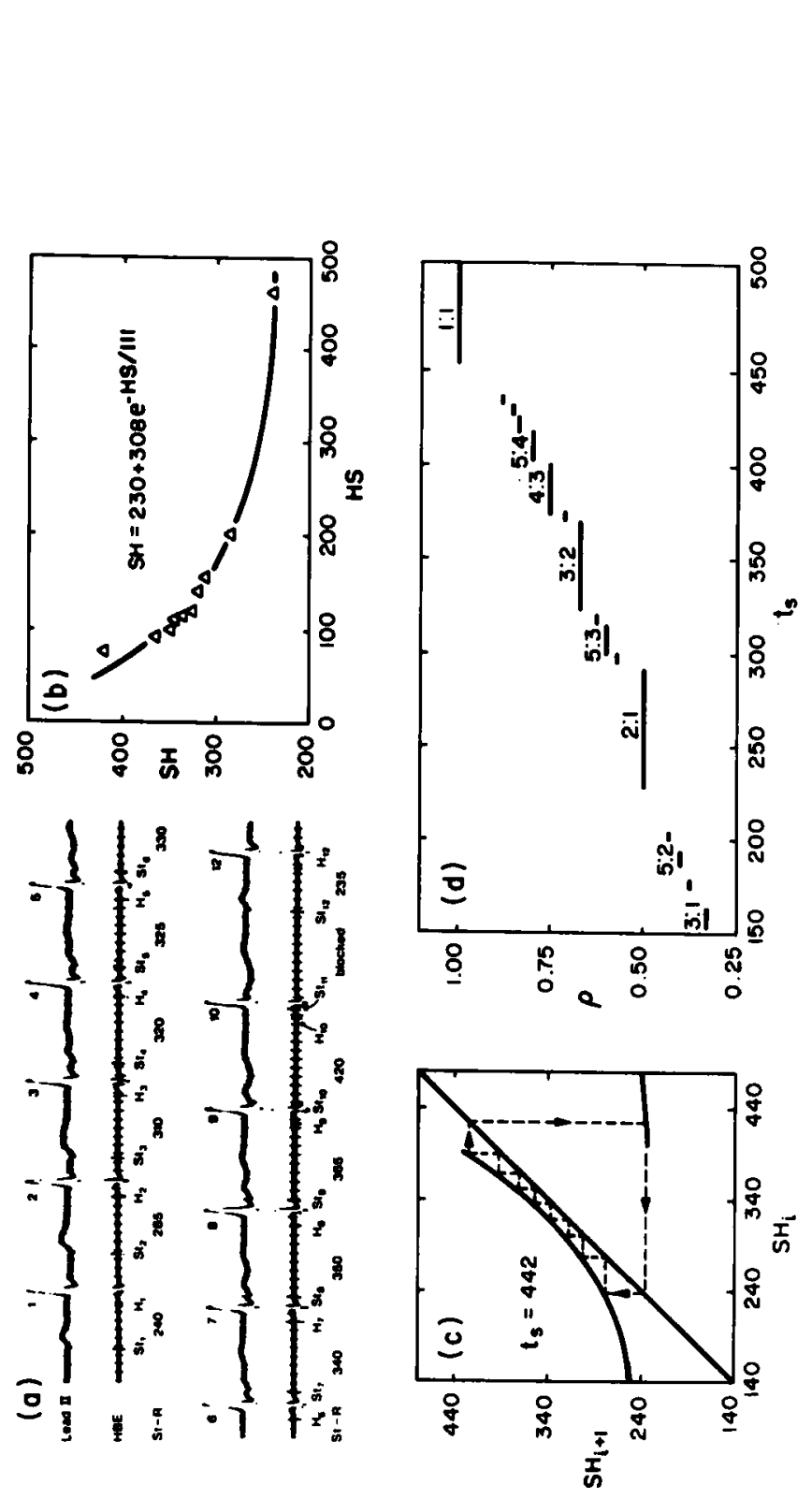

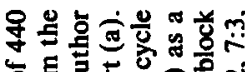

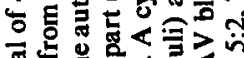

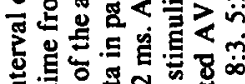

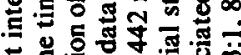
के के

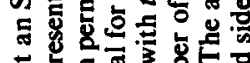

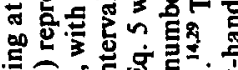

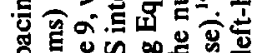

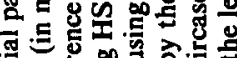

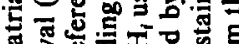

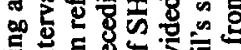

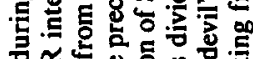

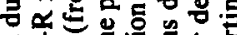

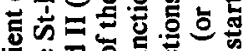

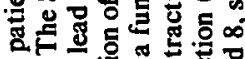
हो.

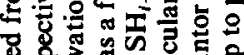

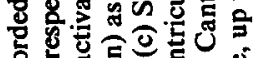
政

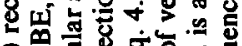
욉 궁

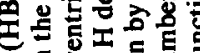

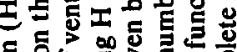

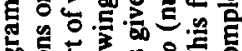

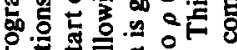
额 政

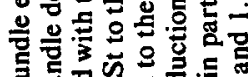

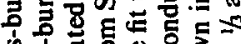

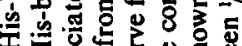

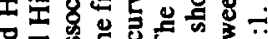

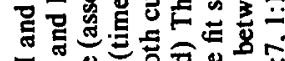
更 于. 을

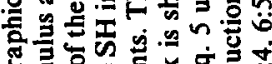

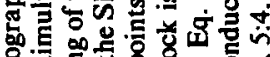
㩆要

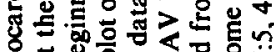

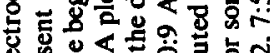

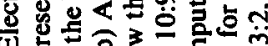

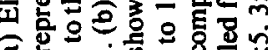
(1)

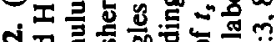
in

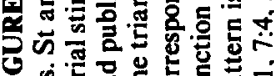

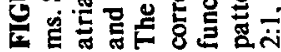


$M / N)$ as a function of $t_{s}$. The function depicted in Figure $2 \mathrm{~d}$ is called a Cantor function or "devil's staircase"; it is a nowhere decreasing function that is piecewise constant on the rationals. ${ }^{14,29}$

The results presented in Figure 2 are of general applicability. Provided that the recovery curve is a monotonically decreasing curve such as shown in FIGURE 2a, then upon periodic stimulation, the same sequence of bifurcations are observed as stimulation frequency is varied. ${ }^{14}$ Thus, for any $N: M(N>M, N$ and $M$ are relatively prime) rhythm, a range of values of $t_{s}$ can be found that generates that rhythm. For example, the typical $N: N-1$ Wenckeback rhythms and $N: 1(N>2)$ high-grade block rhythms, as well as less common variants ${ }^{19,30}$ such as reverse Wenckebach (rhythms intermediate to 5:3 and 2:1) and alternating Wenckebach (rhythms intermediate to 2:1 and $5: 2$ ), and still rarer varieties, should be found at some value of the stimulation frequency. However, as the period of the repeating rhythm increases, the interval of $t_{s}$ over which the rhythm can be observed generally becomes increasingly narrow. Consequently, in experimental systems in which parameters fluctuate, observation of high order periods is difficult, if not impossible, because high order periods will be destroyed by the "noise." The theoretical results provide a firm basis for understanding experimental observations of Wenckebach and allied rhythms in diverse experimental systems. $^{8-10,19,30}$ Tests of the theory have been carried out by measuring the recovery curve of patients and then by using this to predict the conduction ratio as a function of stimulation frequency. These findings confirm that the recovery curve can be used to predict $\mathrm{AV}$ conduction block in humans. ${ }^{31}$

\section{PERIODIC STIMULATION OF SPONTANEOUSLY OSCILLATING CARDIAC TISSUE}

We now consider the effects of periodic stimulation of spontaneously oscillating cardiac tissue. We have conducted extensive studies on aggregates of spontaneously beating cultured ventricular cells isolated from embryonic chick heart. In these experiments, brief current pulses are periodically injected into the aggregate via an intracellular microelectrode. ${ }^{26,27}$ Although we rely on these experiments to illustrate our approach, similar arguments apply in other systems. ${ }^{15-17,23-25}$

As the frequency or amplitude of the periodic stimulation is varied, a large number of different regular and irregular rhythms are observed (some of which are illustrated as insets in FIGURE 3). The patterns include the various Wenckebach and high-grade block rhythms that are found during cardiac arrhythmias. Because the aggregates are essentially electrically homogeneous (space-clamped), the Wenckebach rhythms do not arise from slowing and eventual blocking of conduction. As the stimulation frequency is decreased to a frequency less than the intrinsic beat frequency, escape rhythms $(N: M$ rhythms with $N<M)$ are also observed. One also observes $N: M$ rhythms with $N=M \neq 1$, as well as nonperiodic rhythms. ${ }^{26}$

The origin of this remarkable diversity and the order in which the various rhythms appear as a function of stimulation frequency and amplitude can only be understood from a theoretical analysis of the bifurcations of this system. Assume that the spontaneous cycle length of the oscillator in the absence of periodic forcing is $T_{0}$. 


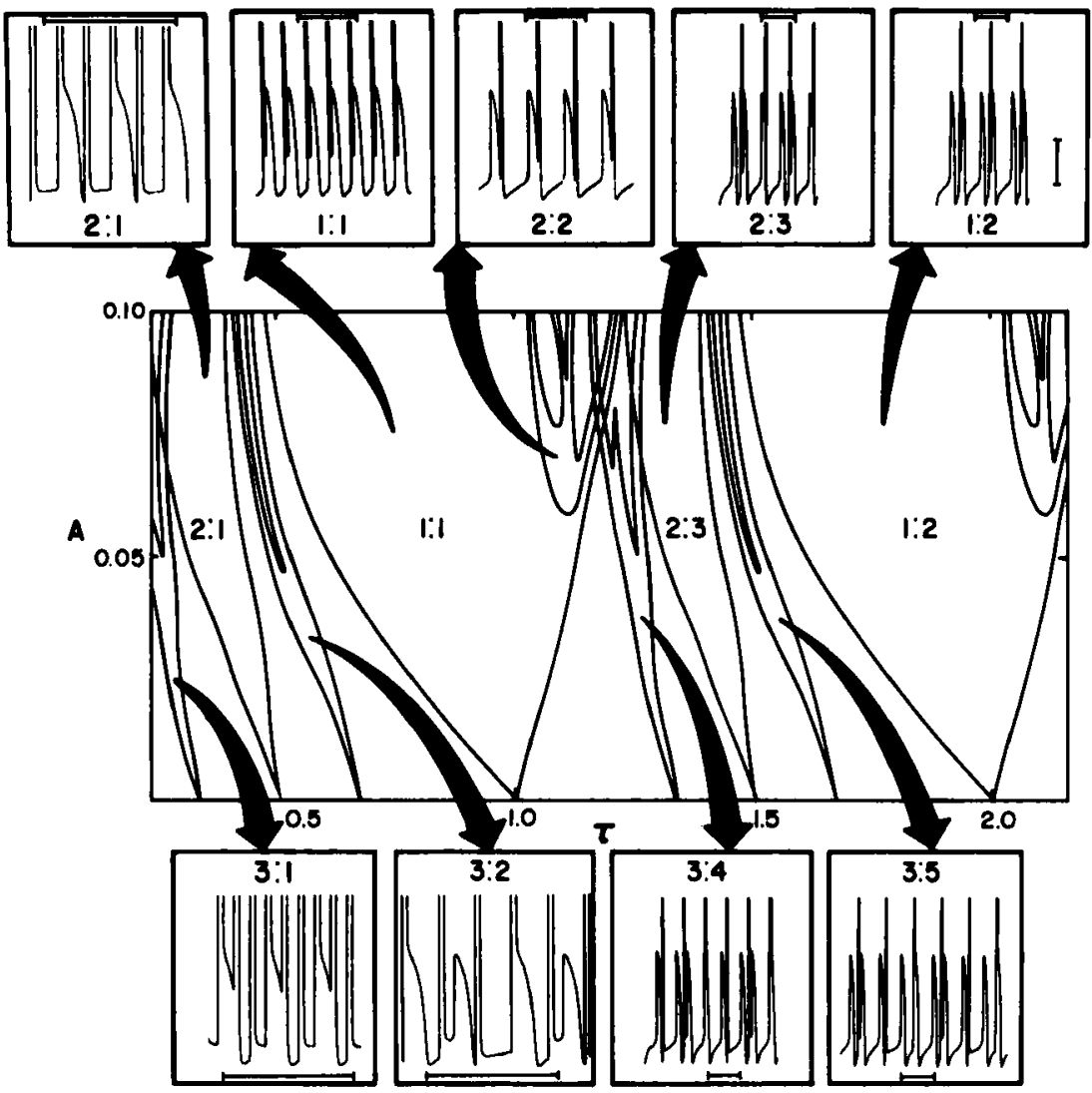

FIGURE 3. Theoretically computed phase-locking zones (solid lines) and illustrative experimental traces (insets) for periodically stimulated aggregates of embryonic chick heart cells. The ordinate is the stimulus amplitude (arbitrary units) ${ }^{27}$ and the abscissa is $\tau-t_{s} / T_{0}$. For $A$ held fixed in the range $0<A<0.039$, the rotation number (the average number of action potentials per stimulus) is theoretically a Cantor function of $\tau$ similar to that shown in FiguRE 2d. For $A>$ 0.039 , the rotation number may theoretically depend on the phase in the cycle at which the first stimulus is delivered. Thus, for these higher stimulus amplitudes, the rotation number for $A$ fixed is not a Cantor function. The theoretically computed curves are taken from reference 27 . Vertical calibration is $50 \mathrm{mV}$ and horizontal calibration is $1 \mathrm{~S}$.

Dividing both sides of Eq. $3 \mathrm{~b}$ by $T_{0}$, we obtain

$$
\phi_{i+1}=F\left(\phi_{i}\right)+\tau(\bmod 1) \text {, }
$$

where

$$
\phi_{i}=\mathrm{RS}_{i} / T_{0}, \quad \tau=t_{s} / T_{0}, \quad F\left(\phi_{l}\right)=-g\left(\phi_{i}, T_{0}\right) / T_{0}(\bmod 1) .
$$

The variable $\phi_{i}$ is the phase $\left(0 \leq \phi_{i}<1\right)$ at which the $i$-th stimulus falls and $F\left(\phi_{i}\right)$ is called the phase transition curve (PTC). ${ }^{25,32}$ The PTC can be determined by delivering a single stimulus pulse at different phases of the cycle. The function that gives the 
percentage deviation of perturbed cycle length from the intrinsic cycle length as a function of phase of stimulus presentation (sometimes called the phase response curve) can be used to compute the PTC. ${ }^{23-28,33}$ In our experiments, we have determined the PTC from data obtained by injecting single puises of various amplitudes. Subsequently, we have fit the PTC to an analytic function and we have computed the ranges of $t_{\text {s }}$ over which stable periodic rhythms (also called phase-locking patterns) are predicted to occur. ${ }^{26,27}$ The results of these computations are shown as the solid lines in Figure 3. Experiments, in general, showed close agreement with the theoretical predictions of FIGURE 3. However, in some regions, for example, $1.05<\tau<1.40,0.06<A<0.10$, where $A$ is an arbitrary stimulus amplitude parameter, ${ }^{27}$ it was difficult to observe the stable patterns that were theoretically predicted to exist over small parameter ranges. Instead, irregular rhythms were observed.

We now consider the bifurcations observed in this system. In the terminology of nonlinear dynamics, Eq. 6a is called a "circle map." ${ }^{34-36}$ This circle map is invertible for low stimulus strengths (less than about $A=0.039$ in our example). All invertible circle maps of the form in Eq. 6a display the same sequence of bifurcations as $\tau$ increases. For this situation, it is known that one must observe the various Wenckebach, high-grade blocks and escape rhythms following the order shown in Figure 3. ${ }^{27.36}$

As the stimulus strength is increased, the PTC computed from our experimental data becomes noninvertible. At the present time, a complete theory for bifurcations in noninvertible circle maps has not been developed. However, it is known that the zones present in the invertible region $(A<0.039)$ will extend into the noninvertible region $(A>0.039) .{ }^{27}$ Also, as a direct consequence of the noninvertibility, zones may branch and cross. This provides a condition in which, even in the absence of noise, either one or the other of two patterns will be seen at the same stimulation frequency and amplitude (bistability), depending on initial conditions. In addition, there can be period-doubling bifurcations in which the period of an oscillation doubles as a parameter changes. These bifurcations lead to alternans, such as the 2:2 rhythms shown in FigurE 3. Finally, the mathematics predicts that there should be chaotic dynamics. We have observed period-doubling bifurcations, "chaotic" dynamics, bistability, and hysteresis (which may be associated with bistability) in our experimental work. ${ }^{26,27,37}$

\section{CONCLUSIONS}

The simple biological and theoretical models that we have described produce rhythms analogous to a large number of different cardiac arrhythmias. These arrhythmias include the simple and complex Wenckebach rhythms, high-grade block, and escape rhythms. ${ }^{8-15}$ Rhythms analogous to parasystolic and related rhythms set up between activity originating in the SA node and in an ectopic focus ${ }^{15-17}$ (e.g., bigeminy with each manifest ectopic beat followed, after a compensatory pause, by a beat of sinus origin) are also observed. Cardiac rhythms demonstrating behavior in which there is alternation of the morphology of one or more of the complexes or of the intervals in the electrocardiogram may be due to period-doubling bifurcations in the heart. ${ }^{36-40}$ Finally, nonperiodic rhythms may be identified with "chaotic" dynamics in the associated models. ${ }^{26,27,36,37,39}$ 
The current work shows that the effect of periodic stimulation of either oscillating or nonoscillating (but excitable) tissue can be understood from an analysis of bifurcations of one-dimensional finite-difference equations containing two or more adjustable parameters. Because the sequences of bifurcations observed do not depend on the detailed functional form of the equations, but rather on their qualitative properties, the bifurcations are "universal."

An interesting aspect of the analysis is that Wenckebach rhythms will arise from periodic stimulation of excitable tissue (whether or not it is spontaneously oscillating) at stimulation rates higher than the intrinsic frequency provided that the stimulation intensity is not too high. Consequently, the appearance of Wenckebach rhythmicity cannot be used to determine whether or not the AV node is spontaneously oscillating, as some suggest, ${ }^{18-21,36}$ or is merely a passive conduit with slow conduction.

The theoretical methods presented in this paper are still at an early stage of development. Several physiological factors such as fatigue of cardiac tissue at high stimulation frequencies, frequency-dependent change of refractory times, and the influence of a blocked action potential on the subsequent conductivity of the AV node are not accounted for in the current formulation of the model. In addition, geometric effects such as spatial variability of refractoriness and conduction velocity in the cardiac muscle also play important roles in the generation of cardiac arrhythmias. Arrhythmias due to reentry, such as some tachycardias and fibrillation, fall outside the scope of the theoretical methods described here. Careful analysis of lengthy rhythm strips of patients is needed to assess the stability of arrhythmias and the bifurcations that occur clinically. Thus, the extent to which the theoretical and biological models have direct applicability to clinical situations is still largely unknown and is a focus for future research. However, the close correspondence between the dynamics in finitedifference equations and those observed clinically strongly suggests that the style of analysis developed above, appropriately modified and extended, will provide a unified theory applicable to a large subset of, but by no means all, cardiac arrhythmias. Consequently, this theoretical approach should be useful in research, teaching, and, perhaps, clinical treatment.

\section{ACKNOWLEDGMENT}

We thank J. Bélair for helpful conversations.

\section{REFERENCES}

1. Chung, E. K. 1977. Principles of Cardiac Arrhythmias. Second Edition. Williams \& Williams. New York.

2. Pick, A. \& R. Langendorf. 1979. Interpretation of Complex Arrhythmias. Lea \& Febiger. Philadelphia.

3. MAY, R. M. 1976. Nature 261: 159-167.

4. Metropolis, N., M. L. Stein \& P. R. Stein. 1973. J. Comb. Theory 15: 25-44.

5. Cvitanovic, P., Ed. 1984. Universality in Chaos. Hilger. Bristol, England.

6. Mines, G. R. 1913. J. Physiol. (London) 46: 349-382.

7. LEWIS, T. \& A. M. MASTER. 1926. Heart 12: 209-270.

8. HeethaAr, R. M., J. J. D. van der Gon \& F. L. MeiJler. 1973. Cardiovasc. Res. 7: $105-114$. 
9. Levy, M. N., P. J. Martin, J. Edelstein \& L. B. Goldberg. 1974. Prog. Cardiovasc. Dis. 16: $601-613$.

10. Simson, M. B., J. Spear \& E. N. MoOre. 1979. Circ. Res. 44: 121-126.

11. MoвitZ, W. 1924. Z. Gesante Exp. Med. 41: 180-237.

12. Decherd, G. M. \& A. Ruskin. 1946. Br. Heart J. 8: 6-16.

13. Smolyaninov, V. V. 1966. Biophysics 11: 382.

14. KeEnER, J. P. 1981. J. Math. Biol, 12: 215-225.

15. JaLiFe, J. \& D. C. MiChaELS. 1985. In Cardiac Electrophysiology and Arrhythmias. D. P. Zipes \& J. Jalife, Eds.: 109-119. Grune \& Stratton. New York.

16. JALIFE, J. \& G. K. MOE. 1976. Circ. Res. 39: 801-808.

17. JALIFE, J. \& G. K. MOE. 1979. Am. J. Cardiol. 43: 761-772.

18. VAN DER POL, B. \& J. VAN DER Mark. 1928. Philos. Mag. 6: 763-775.

19. Roberge, F. A. \& R. A. Nadeau. 1969. Can. J. Physiol. Pharmacol. 47: 695-704.

20. Sideris, D. A. \& S. D. MOULOPOULOS. 1977. J. Electrocardiol. 10: 51-58.

21. West, B. J., A. L. Goldberger, G. Rovner \& V. Bhargava. 1985. Physica 17D: 198206.

22. LeVI, M. 1981. Mem. Am. Math. Soc. 32: no. 244.

23. Perkel, D. H., J. H. Schulman, T. H. Bullock, G. P. Moore \& J. P. Segundo. 1964. Science 145: 61-63.

24. Pittendrigh, C. S. 1965. In Circadian Clocks. J. Aschoff, Ed.: 277-297. North-Holland. Amsterdam.

25. Pavlidis, T. 1973. Biological Oscillations: Their Mathematical Analysis. Academic Press. New York.

26. Guevara, M. R., L. Glass \& A. Shrier. 1981. Science 214: 1350-1353.

27. Glass, L., M. R. Guevara, J. Bélair \& A. Shrier. 1984. Phys. Rev. 29A: 1348-1 357.

28. Honer KAMP, J. 1983. J. Math. Biol. 18: 69-88.

29. Mandelbror, B. B. 1982. The Fractal Geometry of Nature. Freeman. San Francisco.

30. Halpern, M. S., G. J. Nau, R. J. Levi, M. V. Elizari \& M. B. Rosenbaum. 1973. Circulation 48: 41-49.

31. Shrier, A., H. Dubarsky, M. Rosengarten, M. R. Guevara, S. Nattel \& L. Glass. 1987. Submitted.

32. KaWATO, M. 1981. J. Math. Biol. 12: 13-30.

33. Winfree, A. T. 1980. The Geometry of Biological Time. Springer Pub. New York.

34. Glass, L. \& R. Perez. 1982. Phys. Rev. Lett. 48: 1772-1775.

35. Feigenbaum, M. J., L. P. Kadanoff \& S. J. Shenker. 1982. Physica 5D: 370-386.

36. Guevara, M. R. \& L. Glass. 1982. J. Math. Biol. 14: 1-23.

37. Glass, L., A. Shrier \& J. Bélair. 1986. In Chaos. A. V. Holden, Ed.: 237-256. Manchester Univ. Press. Manchester, England.

38. Guevara, M. R., G. Ward, A. Shrier \& L. Glass. 1984. Computers in cardiology. IEEE Comp. Soc., p. 167-170.

39. RitzenberG, A. L., D. R. ADAm \& R. J. COHeN. 1984. Nature 307: 159-161.

40. Goldberger, A. L., R. Shabetal, V. Bhargarva, B. J. West \& A. J. Mandell. 1984. Am. Heart J. 107: 1297-1299.

\section{DISCUSSION OF THE PAPER}

J. ARON: You commented that even if you made an extremely complicated, detailed model, you would end up showing how useful the simple difference equation was. Do you think, then, that one should go ahead and build detailed models?

L. GLASs: That is a good point. I think that if you do build them, then you are going to see the same sorts of things. In the intact patient, things are bound to be more complicated than the simple bifurcations we are seeing here. I should have stated at the 
outset that these are not all the arrhythmias in the heart. There are arrhythmias with important propagation effects, which indeed must be analyzed with models that include propagation, and $\mathbf{l}$ am sure that effects can be seen with propagation that are not present in these models. When somebody starts doing complicated models and more realistic geometries, that person is going to see the sort of stuff that is happening in our model, that is, the first-order and maybe the second-order things going on. It is interesting from a theoretical point of view that those complicated things are showing the same sorts of bifurcations that we have here. I think that there is a lot of value in trying to take the next steps in building the models.

M. SHLESINGER: Could you make any comments about ionic mechanisms? Are there any general statements (such as, you would need the calcium besides the sodium and potassium currents)? Do you need a couple of differential equations and, say, more than two variables?

GLASS: To my knowledge, the people who have tried to look at phase resetting using ionic mechanisms would include Guevara, Clay, Shrier, and others. The ionic mechanisms that they have examined give reasonable agreement with most of the features observed experimentally. With the ionic model, you need at least two variables to get an oscillation. Most of those who look at the ionic models try to use the most accurate equations available now and try to do the computer experiments on the models. I cannot make any stronger generalizations.

E. BASAR: During these arrhythmias, did you try to observe a kind of competition between various rhythms from various parts of the heart?

Glass: Yes. In Michael Guevara's thesis and also in some papers submitted for publication, over certain regions of the parameter space we showed the presence of $2: 1$ and $1: 1$ rhythms at the same stimulation parameters and demonstrated a hysteresis effect. Also, in the regions where the Arnol'd tongues overlap one another, there are extremely complicated dynamics, and one potential interpretation is that there may be two basins of attraction in addition to the noise. Our discussion of that is in a paper called "Chaotic Cardiac Rhythms," which is in Arun Holden's recent collection of papers on chaos. ${ }^{37}$

I think there is evidence for bistability. Different sorts of bistability can arise in different regions of the parameter space. It is a very delicate operation to observe it in this preparation (at least in the way that we have tried to do it), but it does exist.

BASAR: We observed something similar in the brain rhythms, but it is very difficult to interpret. The heart is, I guess, a simpler system. If we start to learn something there, then we can take the ideas to the brain.

GLASS: Only somebody who studies the brain would say that the heart is simpler than the brain. 